\title{
Quantized Predictive Control over Erasure Channels
}

\author{
Daniel E. Quevedo, Member, IEEE, and Jan Østergaard, Member, IEEE
}

\begin{abstract}
We study a control architecture for linear timeinvariant plants which are affected by random disturbances. The distinguishing aspect of the situation at hand is that an unreliable data-rate limited network is placed between controller and the plant input. To achieve robustness with respect to i.i.d. dropouts, the controller transmits data packets containing quantized plant input predictions. These minimize a finite horizon cost function and are provided by an appropriate optimal entropy coded dithered lattice vector quantizer. Within this context, we derive an equivalent noise-shaping model of the closed loop system. This model is employed for the design and analysis of the vector quantizer used by the controller.
\end{abstract}

\section{INTRODUCTION}

The interest in closed loop control over communication networks has increased tremendously in recent years; see, e.g., [1]-[3] and the references therein. In particular, Ethernet in its wired (hub-based and switched) and wireless forms (IEEE 802.11) is increasingly being adopted as a low level control network technology, see [4], [5]. The reasons for this move towards Networked Control Systems (NCS's) are manifold, including lower cost, higher reliability, interoperability of devices, and easier installation and maintenance.

From a control design perspective, many interesting challenges are associated with NCS's. For example, due to the inherent bit-rate limitations associated with digital networks, signals need to be coded and quantized prior to transmission [2]. Furthermore, the network may induce variable delays and data-dropouts [1]. It turns out that, in a NCS, links are not transparent, often constituting a significant bottleneck in the achievable performance. Successful NCS design methods need to consider both control and communication aspects.

For the design of NCS's one can often take advantage of the fact that in contemporary communication networks, data is sent in large time-stamped packets. ${ }^{1}$ This motivates the development of control methods in which packets of signal predictions are sent. These are calculated at the transmission side to compensate for random time delays and packetdropouts, are sent. At the receiver node, packets are buffered and only the latest relevant value is used. In particular, the idea of sending packets with plant input predictions obtained via predictive control formulations (hereafter termed packetized predictive control (PPC)) was proposed in [6] for the

Daniel Quevedo is with the School of Electrical Engineering \& Computer Science, The University of Newcastle, NSW 2308, Australia; e-mail: dquevedo@ieee.org. Jan Østergaard is with the Department of Electronic Systems, Aalborg University, Denmark; e-mail: janoe@ieee.org

This research was supported under Australian Research Council's Discovery Projects funding scheme (project number DP0988601).

${ }^{1}$ For example, the frame format in IP based Ethernet allows for a datapacket of 46-1500 bytes, the overhead being 26 bytes. Time-stamping can be easily carried out, e.g., by invoking the Real Time Transport Protocol.

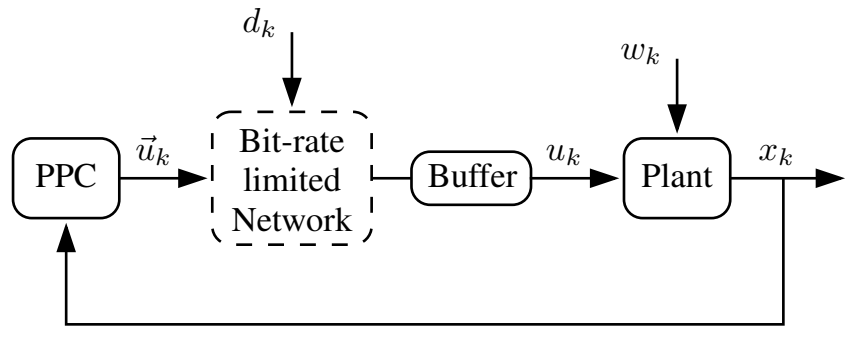

Fig. 1. NCS Configuration

teleoperation of prestabilized constrained nonlinear plants. The concept also underlies more recent NCS configurations described, e.g., in [7]-[14]. Experimental results of NCS's which use PPC are promising. However, only limited supporting theoretical results exist, specially for the case of NCS's with disturbances, see [7], [11]. To the best of the authors' knowledge, no works on PPC have treated bit-rate limitations, or, equivalently, quantization issues.

The present paper studies a PPC scheme for discretetime linear time invariant plants with random disturbances. Signal transmission is over a bit-rate limited network which is affected by i.i.d. random packet-dropouts, see Fig. 1. The controller respects bit-rate constraints of the network by sending optimally quantized finite-horizon plant input sequences. These are provided by an appropriate entropy constrained lattice vector quantizer. Within this context, we develop analytical expressions for the power spectral densities of the signals of interest. Through use of an equivalent noise-shaping model of the NCS, we obtain results on optimal lattice vector quantization as used by the controller.

The remainder of this paper is organized as follows: In Section II we present the NCS under study. Section III carries out closed loop analysis. Results on the PPC are included in Section IV. Section V draws conclusions.

Notation: I denotes the identity matrix, where the dimension follows from the context; the superscript ${ }^{T}$ denotes transpose, whereas ${ }^{H}$ refers to Hermitian transposition of a matrix; $e_{1}^{T}=\left[\begin{array}{llll}1 & 0 & \cdots & 0\end{array}\right] \in \mathbb{R}^{1 \times N}$. For any matrix $K$, $\operatorname{det} K$ denotes its determinant, $\operatorname{tr} K$ its trace; $K \succeq 0$ means that $K$ is positive semi-definite, $\succ$ refers to positive-definite. For any positive semi-definite matrix $P$ and any vector $\xi$, we define $\|\xi\|_{P}^{2} \triangleq \xi^{T} P \xi$. For any asymptotically stationary stochastic processes $\zeta=\left\{\zeta_{k}\right\}$ and $\eta=\left\{\eta_{k}\right\}$, we define the covariance matrices $R_{\zeta \eta}(\ell) \triangleq \lim _{k \rightarrow \infty} \mathcal{E}\left\{\zeta_{k} \eta_{k+\ell}^{T}\right\}$ and $R_{\zeta}(\ell) \triangleq \lim _{k \rightarrow \infty} \mathcal{E}\left\{\zeta_{k} \zeta_{k+\ell}^{T}\right\}$, where $\mathcal{E}(\cdot)$ is the expectation operator. If $\zeta$ is zero-mean Gaussian distributed with variance $R_{\zeta}(0)$, then we write $\zeta \sim \mathcal{N}\left(0, R_{\zeta}(0)\right)$. 


\section{Packetized Quantized Predictive Control OVER ERASURE CHANNELS}

We consider a discrete-time linear time invariant (LTI) plant model with state $x_{k} \in \mathbb{R}^{n}, n \geq 1$ and scalar input $u_{k} \in \mathbb{R}:$

$$
x_{k+1}=A x_{k}+B_{1} u_{k}+B_{2} w_{k} .
$$

In (1), $w_{k} \in \mathbb{R}^{m}$, where $m \geq 1$, is an unmeasured disturbance which is modelled as an arbitrarily distributed i.i.d. zero-mean stochastic process with power spectral density (PSD) $S_{w}\left(e^{j \omega}\right)$ and covariance matrix $R_{w}(0)$.

\section{A. Network Effects}

Our interest lies in clock-driven Ethernet-like networks situated between controller output and plant input. Thus, all data to be transmitted needs to be quantized and is sent in large time-stamped packets. Due to transmission errors and congestion, the network introduces packet-dropouts and time-delays. From a closed-loop control perspective, packetdropouts are the most serious network effect. This motivates us to model the network as an erasure channel, which operates at the same sampling rate as the plant model (1), see also [15], [16] ${ }^{2}$. In the sequel, we model transmission effects via the discrete Bernoulli process $\left\{d_{k}\right\}$ defined via:

$d_{k} \triangleq \begin{cases}1 & \text { if packet-dropout occurs at instant } k, \\ 0 & \text { if packet-dropout does not occur at instant } k .\end{cases}$

We will assume that each variable $d_{k}$ is i.i.d. with distribution

$$
\operatorname{Prob}\left(d_{k}=1\right)=p, \quad \operatorname{Prob}\left(d_{k}=0\right)=1-p,
$$

where $p \in(0,1)$ is the dropout-rate.

As foreshadowed in the introduction, at each time instant $k$, the PPC sends a control packet, say $\vec{u}_{k}$, to the plant input node. To achieve good performance, despite unreliable communication, $\vec{u}_{k}$ contains possible control inputs for a finite number of $N$ future time instants. At the plant input side, the received packets are buffered, providing the plant inputs, see Fig. 1. In what follows, we will first describe the buffering procedure and then the control packet design.

\section{B. Buffering}

The buffering mechanism amounts to a parallel-in serialout shift register, which acts as a safeguard against packetdropouts. For that purpose, the buffer state, say $b_{k} \in \mathbb{R}^{N}$, is overwritten whenever a valid (i.e., uncorrupted and undelayed) control packet arrives. Actuator values are passed on to the plant sequentially until the next valid control packet is received, see Fig. 1. More formally, we have:

$$
\begin{aligned}
b_{k} & =d_{k} M b_{k-1}+\left(1-d_{k}\right) \vec{u}_{k}, \\
u_{k} & =e_{1}^{T} b_{k}
\end{aligned}
$$

\footnotetext{
${ }^{2}$ Note that small fixed time-delays up to a fixed threshold can be included in (1). Signals, which are delayed more, are then considered as "lost."
}

where

$$
M \triangleq\left[\begin{array}{ccccc}
0 & 1 & 0 & \ldots & 0 \\
\vdots & \ddots & \ddots & \ddots & \vdots \\
0 & \ldots & 0 & 1 & 0 \\
0 & \ldots & \ldots & 0 & 1 \\
0 & \ldots \ldots & \ldots & \ldots & 0
\end{array}\right] \in \mathbb{R}^{N \times N}
$$

is a shift matrix. The initial buffer state is taken as $b_{0}=0$.

\section{Control Packet Design}

The control packets $\vec{u}_{k}$ are formed using a predictive control approach. At each time instant $k$ and for a given plant state $x_{k},{ }^{3}$ the following cost function is minimized:

$$
J\left(\vec{u}^{\prime}\right) \triangleq\left\|x_{N}^{\prime}\right\|_{P}^{2}+\sum_{\ell=0}^{N-1}\left\|x_{\ell}^{\prime}\right\|_{Q}^{2}+\lambda\left(u_{\ell}^{\prime}\right)^{2},
$$

where $P, Q \succeq 0, \lambda>0$ and $N \geq 1$ are design variables.

The cost function in (3) examines predictions of the nominal system over a finite horizon of length $N$. In particular, the predicted state trajectories are generated by:

$$
x_{\ell+1}^{\prime}=A x_{\ell}^{\prime}+B_{1} u_{\ell}^{\prime}, \quad x_{0}^{\prime}=x_{k}
$$

whilst the entries in the vector $\vec{u}^{\prime}=\left[\begin{array}{lll}u_{0}^{\prime} & \ldots & u_{N-1}^{\prime}\end{array}\right]^{T}$ are the associated plant inputs.

Following the ideas underlying PPC, see, e.g., [12], at each time instant $k$, and for associated state $x_{k}$, the controller sends an optimizing packet $\vec{u}_{k}$ to the actuator node. This procedure is repeated ad infinitum.

\section{Quantization Constraints}

A distinguishing aspect of the situation at hand is that the network is bit-rate limited. Hence, data transmitted through the network needs to be quantized. This introduces a quantization constraint into the problem of minimizing $J\left(\vec{u}^{\prime}\right)$. For the case of linear systems with a quadratic cost function, the following result, taken from [17], characterizes its solution:

Theorem 1 (Quantized Predictive Control [17]):

Consider any quantized set $\mathbb{U} \subset \mathbb{R}^{N}$, the matrices $\Gamma$ and $\Psi$ given in Appendix A, and define:

$$
\xi_{k} \triangleq \Gamma x_{k} .
$$

Then the optimizer $\vec{u}_{k}=\arg \min _{\vec{u}^{\prime} \in \mathbb{U}} J\left(\vec{u}^{\prime}\right)$, see (3), satisfies:

$$
\vec{u}_{k}=\Psi^{-1} q\left(\xi_{k}\right),
$$

where $q(\cdot)$ is a (nearest neighbour) vector quantizer with alphabet $\Psi \mathbb{U}$.

Proof: The proof resembles that of Thm. 1 in [17].

Theorem 1 forms the basis of the present work. It shows that designing a quantized PPC for the system (1) is equivalent to designing a vector quantizer for $\xi_{k} .^{4}$ This amounts to designing the alphabet $\Psi \mathbb{U}$. In Section IV we will show

\footnotetext{
${ }^{3}$ We will consider a situation where there are no acknowledgments. Thus, the controller does not have certainty about whether previous packets have arrived at the plant side, compare to recent work in [11], [14].

${ }^{4}$ See [18] for more background on vector quantizers.
} 
how to optimize NCS performance through design of the quantizer alphabet.

It is worth noting that in [17] the quantizer alphabet $\mathbb{U}$ was given, whereas here $\mathbb{U}$ constitutes an important design parameter. Quantization constraints arise due to the need to accommodate the fact that the network is bit-rate limited.

Remark 1 (Pre-and Post-filtering): It is well-known that it is often useful to include pre- and post-filtering around the quantizer, see, e.g., [19]-[22]. How to design optimal preand post-filters for closed loop applications has been treated partially in the above references. We note that any causal LTI filtering can be easily incorporated into our framework by augmenting the plant model (1) with the post-filter. The pre-filter is implicit in the controller.

\section{Closed Loop AnAlysis}

We have seen in the previous section that the packetized predictive controller comprises a vector quantizer $q(\cdot)$. As will become apparent in Section IV, this quantizer will be chosen to satisfy the additive noise model (see, e.g., [26])

$$
q\left(\xi_{k}\right)=\xi_{k}+n_{k}
$$

where the quantization noise $\left\{n_{k}\right\}$ is independent of $\left\{w_{k}\right\}$. In addition, $n_{k}$ is independent of $\xi_{k-\ell}, \forall \ell \geq 0$. For further reference, we will use $S_{n}\left(e^{j \omega}\right)$ to denote the PSD of $\left\{n_{k}\right\}$.

\section{A. Mean Square Stability}

Given (1)-(6), the NCS can be characterized via:

$$
\begin{aligned}
& x_{k+1}=A x_{k}+B_{1} e_{1}^{T} b_{k}+B_{2} w_{k} \\
& b_{k+1}=d_{k+1} M b_{k}+\left(1-d_{k+1}\right) \Psi^{-1}\left(\Gamma x_{k+1}+n_{k+1}\right),
\end{aligned}
$$

or, equivalently, via:

$$
\theta_{k+1}=\bar{A}\left(d_{k+1}\right) \theta_{k}+\bar{B}\left(d_{k+1}\right) \nu_{k}
$$

where:

$$
\theta_{k} \triangleq\left[\begin{array}{c}
x_{k} \\
b_{k}
\end{array}\right], \nu_{k} \triangleq\left[\begin{array}{c}
w_{k} \\
n_{k+1}
\end{array}\right]
$$

and where:

$$
\begin{gathered}
\bar{A}(0)=\left[\begin{array}{cc}
A & B_{1} e_{1}^{T} \\
\Psi^{-1} \Gamma A & \Psi^{-1} \Gamma B_{1} e_{1}^{T}
\end{array}\right], \bar{A}(1)=\left[\begin{array}{cc}
A & B_{1} e_{1}^{T} \\
0 & M
\end{array}\right], \\
\bar{B}(0)=\left[\begin{array}{cc}
B_{2} & 0 \\
\Psi^{-1} \Gamma B_{2} & \Psi^{-1}
\end{array}\right], \bar{B}(1)=\left[\begin{array}{cc}
B_{2} & 0 \\
0 & 0
\end{array}\right] .
\end{gathered}
$$

Thus, the NCS constitutes a Markov jump-linear system, whose properties have been studied, e.g., in [23]. In particular, the following theorem gives necessary and sufficient conditions for stationarity and mean-square stability (MSS):

Theorem 2 (Stationarity and MSS; adapted from [23]):

The system in (9) is MSS and asymptotically wide-sense stationary, if and only if there exists $\Xi \succ 0$ such that

$$
\Xi-(1-p) \bar{A}^{T}(0) \Xi \bar{A}(0)-p \bar{A}^{T}(1) \Xi \bar{A}(1) \succ 0 .
$$

Proof: Follows from results in Chapter 3 of [23].

\section{B. Power Spectral Densities}

A key element of the present work lies in the design and analysis of the vector quantizer $q(\cdot)$ in (5). For that purpose, we will use the PSDs provided by the following theorem:

Theorem 3 (PSDs): Suppose that the system (9) is stable and stationary and define:

$$
\begin{aligned}
G_{1}\left(e^{j \omega}\right) & \triangleq\left(e^{j \omega} I-A\right)^{-1} B_{1} e_{1}^{T}, \\
G_{2}\left(e^{j \omega}\right) & \triangleq\left(e^{j \omega} I-A\right)^{-1} B_{2}, \\
T\left(e^{j \omega}\right) & \triangleq(1-p)\left(I-p M e^{j \omega}\right)^{-1} .
\end{aligned}
$$

Then the (asymptotic) PSDs of $x, b$ and $u$ satisfy:

$$
\begin{aligned}
& S_{x}\left(e^{j \omega}\right)=F_{n}\left(e^{j \omega}\right) S_{n}\left(e^{j \omega}\right) F_{n}^{H}\left(e^{j \omega}\right) \\
& +F_{w}\left(e^{j \omega}\right) S_{w}\left(e^{j \omega}\right) F_{w}^{H}\left(e^{j \omega}\right), \\
& S_{b}\left(e^{j \omega}\right)=E_{n}\left(e^{j \omega}\right) S_{n}\left(e^{j \omega}\right) E_{n}^{H}\left(e^{j \omega}\right) \\
& +E_{w}\left(e^{j \omega}\right) S_{w}\left(e^{j \omega}\right) E_{w}^{H}\left(e^{j \omega}\right), \\
& S_{u}\left(e^{j \omega}\right)=e_{1}^{T} S_{b}\left(e^{j \omega}\right) e_{1},
\end{aligned}
$$

where:

$$
\begin{aligned}
& F_{n}\left(e^{j \omega}\right) \triangleq H_{x}\left(e^{j \omega}\right) G_{1}\left(e^{j \omega}\right) T\left(e^{j \omega}\right) \Psi^{-1} \\
& F_{w}\left(e^{j \omega}\right) \triangleq H_{x}\left(e^{j \omega}\right) G_{2}\left(e^{j \omega}\right) \\
& E_{n}\left(e^{j \omega}\right) \triangleq T\left(e^{j \omega}\right) \Psi^{-1}\left(I+\Gamma F_{n}\left(e^{j \omega}\right)\right) \\
& E_{w}\left(e^{j \omega}\right) \triangleq T\left(e^{j \omega}\right) \Psi^{-1} \Gamma F_{w}\left(e^{j \omega}\right) \\
& H_{x}\left(e^{j \omega}\right) \triangleq\left(I-G_{1}\left(e^{j \omega}\right) T\left(e^{j \omega}\right) \Psi^{-1} \Gamma\right)^{-1} .
\end{aligned}
$$

Proof: See Appendix B.

The above result allows us to characterize performance of the packetized NCS in the presence of quantization, random data-dropouts and disturbances. It constitutes the main contribution of this section.

For further reference, we define the matrices:

$$
\begin{aligned}
& M_{n}=\frac{1}{2 \pi} \int_{0}^{2 \pi} F_{n}\left(e^{j \omega}\right) F_{n}^{H}\left(e^{j \omega}\right) d \omega \\
& M_{w}=\frac{1}{2 \pi} \int_{0}^{2 \pi} F_{w}\left(e^{j \omega}\right) S_{w}\left(e^{j \omega}\right) F_{w}^{H}\left(e^{j \omega}\right) d \omega .
\end{aligned}
$$

\section{Design AND Analysis of PPC Via Vector QUANTIZATION TECHNIQUES}

We will next use the insights gained by Theorem 3 for the design and analysis of PPC over bit-rate limited erasure channels. Here, we will exploit the fact that, as shown in Theorem 1, the PPC can be regarded as a specific vector quantizer (VQ). Thus, PPC design becomes VQ design.

As mentioned in Section II, we are interested in the socalled UDP-like case where there are no receipt acknowledgments. In order to guarantee that at the plant input side successful decoding of $q\left(\xi_{k}\right)$ can be achieved despite random data-loss, we will use a memoryless VQ. More specifically, we will limit our attention to (entropy-coded) dithered lattice VQs, hereafter abbreviated ECDQs.

Remark 2 (Optimal VQs): For any finite dimension $N$ and bit-rate, it is known that, optimal entropy-constrained 
VQs minimize the expected distortion. These VQs can be designed, e.g., via the LBG algorithm [18]. Unfortunately, the resultant quantizers usually lack structure and, thus, search complexity is substantial. Furthermore, redesign is required for different bit-rates. On the contrary, lattice VQs have fast implementations and often give near optimal performance, making them a practical alternative; see, e.g., [24].

\section{A. Entropy Constrained Dithered Lattice VQs}

An ECDQ uses subtractive dither, where the dither is a random zero-mean i.i.d. vector process. The latter is known to the encoder as well as the decoder; see, e.g., [25]. The current dither vector is independent of current and past values of the input signal and is uniformly distributed over the basic Voronoi cell of the lattice quantizer. It follows that the quantization error $n_{k}$ in (6) is statistically independent of the current and past values of the input signal $\xi_{k}$. Furthermore, each $n_{k}$ is a zero-mean white vector with variance

$$
\sigma_{n}^{2}=\operatorname{tr} R_{n}(0)
$$

see also [26].

\section{B. ECDQ Design}

For a given dimension $N$ and lattice-type $\Lambda_{N}$, see [27], designing an ECDQ amounts to finding an appropriate value of the quantization noise variance $\sigma_{n}^{2}$. In fact, we have:

$$
\sigma_{n}^{2}=N \mathcal{G}\left(\Lambda_{N}\right) V^{2 / N}
$$

where $V$ is the volume of the Voronoi cell and $\mathcal{G}\left(\Lambda_{N}\right)$ is its dimensionless normalized second moment of inertia, see [26]. The latter is fixed for a given lattice. Thus, $\sigma_{n}^{2}$ determines $V$ and thereby completely specifies the quantizer.

In accordance with the control objective in (3), we will adopt the distortion measure given below as a figure of merit of the PPC quantization system:

$$
D \triangleq \operatorname{tr}\left(Q^{1 / 2} R_{x}(0) Q^{T / 2}\right)+\lambda R_{u}(0)
$$

where $Q=Q^{T / 2} Q^{1 / 2}$. This distortion measure quantifies loss of performance of the NCS due to quantization and packet dropouts. Interestingly, we have the following result:

Lemma 1: The distortion measure in (17) is affine in $\sigma_{n}^{2}$, i.e., there exist $\alpha$ and $\beta$, such that:

$$
D=\alpha \sigma_{n}^{2}+\beta, \quad \alpha, \beta>0 .
$$

Proof: Use of (14), (15) and Parseval's Theorem gives:

$$
R_{x}(0)=\left(\sigma_{n}^{2} / N\right) M_{n}+M_{w},
$$

which is affine in $\sigma_{n}^{2}$. A similar result can be established for $R_{u}(0)$ from (12) and (13). Linearity of the trace operator then proves our result.

As a consequence of this lemma, for a given distortion level $D$, we can uniquely determine $\sigma_{n}^{2}$. This defines the quantizer and, thus, the controller. Note that the scalars $\alpha$ and $\beta$ in (18) are independent of the quantizer parameters, but depend upon the dropout-rate $p$. Hence optimal quantizer design requires knowledge of the dropout-rate.

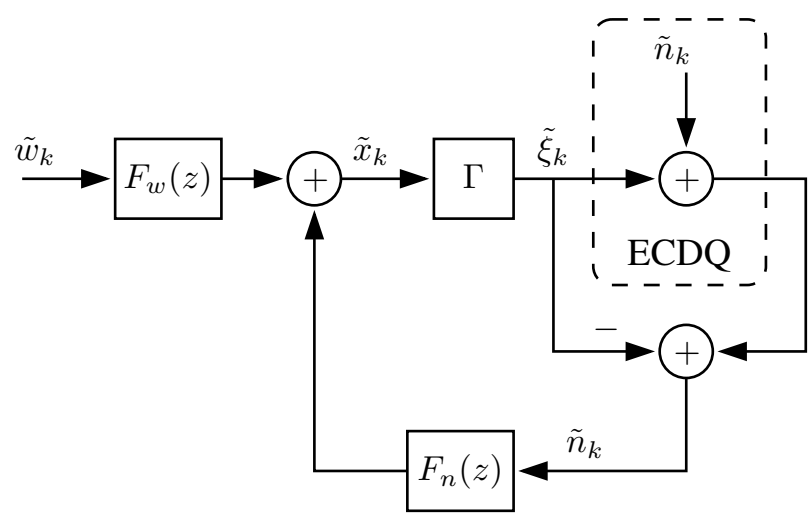

Fig. 2. Noise-Shaping Model of the NCS

\section{Rate Bound}

Since we consider a NCS which uses a data-rate limited network, it is important to establish a relationship between the coding rate, i.e., the average bit-rate of the quantizer output and the achieved distortion $D$, see (17). For the situation at hand, the characterization of the PSDs obtained in Theorem 3, which is summarized in the following corollary, is of fundamental importance. ${ }^{5}$

Corollary 1: The PSD of $\tilde{x}_{k}$ in the noise-shaping model depicted in Fig. 2 is equal to the PSD of $x_{k}$ in Fig. 1.

Proof: Follows immediately from (4), (6) and (11).

The equivalent noise-shaping structure provided by Fig. 2 allows us to analyze the jump-linear system (9) by adapting techniques for LTI coding systems. In particular, Theorem 4 included below establishes an upper bound on the coding rate. Our result is valid at all rates and, thus, does not require high-rate approximations.

Theorem 4 (Rate Bound): For any $1 \leq N \in \mathbb{N}$, the coding rate $R$ of $\vec{u}_{k}$ satisfies:

$R \leq \frac{1}{2} \log _{2}\left(\operatorname{det}\left(I+\left(N / \sigma_{n}^{2}\right) R_{\xi}(0)\right)\right)+\frac{N}{2} \log _{2}\left(\frac{\pi e}{6}\right)+1$,

where $\xi$ is as in (4).

Proof: See Appendix C.

\section{Constraints arising from Quantization Noise}

The rate-bound (20) motivates us to introduce the SNRtype quantizer performance ratio:

$$
\gamma \triangleq \frac{\operatorname{det} R_{\xi}(0)}{\sigma_{n}^{2}}
$$

The quantization noise variance $\sigma_{n}^{2}$ can be arbitrarily chosen. Interestingly, due to feedback of $n_{k}$, provided the closed loop is stationary, $\gamma$ always satisfies the bound established in the following lemma: ${ }^{6}$

\footnotetext{
${ }^{5}$ Note that a related equivalence was established in [28] for a simpler non-packetized NCS with dropouts.

${ }^{6}$ Compare to SNR constraints used in the context of NCS's where transmitted signals are scalar; see, e.g., [20], [21], [28].
} 
Lemma 2 (Bound on $\gamma$ ): Suppose that (21) holds and that the loop in Fig. 1 is asymptotically stationary. Then

$$
\gamma \geq \max _{i} \nu_{i}
$$

where $\left\{\nu_{i}\right\}$ are the eigenvalues of the matrix

$$
\Theta \triangleq \frac{\operatorname{det} R_{\xi}(0)}{N} \Gamma M_{n} \Gamma^{T} R_{\xi}^{-1}(0) .
$$

Proof: See Appendix D.

\section{CONCLUSions}

The present work has studied a packetized predictive control strategy for a control architecture comprising an LTI plant with disturbances and a bit-rate limited digital network affected by i.i.d. packet dropouts. We have characterized the power spectral densities of key signals in the loop and have developed an equivalent noise-shaping model. The insights gained has allowed us to design and analyze the controller as an optimal entropy coded dithered lattice vector quantizer. Future work could include further analysis of closed loop stability in the presence of data-dropouts and quantization.

\section{APPENDIX}

\section{A. Matrices used in Theorem 1}

In (4), the gain $\Gamma$ is given by:

$$
\Gamma=-\Psi^{-T} \Phi^{T} \bar{Q} \Lambda
$$

where $\bar{Q} \triangleq \operatorname{diag}(Q, \ldots, Q, P) \in \mathbb{R}^{N n \times N n}$ and where:

$\Phi \triangleq\left[\begin{array}{ccccc}B_{1} & 0 & \ldots & 0 & 0 \\ A B_{1} & B_{1} & \ldots & 0 & 0 \\ \vdots & \vdots & \ddots & \vdots & \vdots \\ A^{N-1} B_{1} & A^{N-2} B_{1} & \ldots & A B_{1} & B_{1}\end{array}\right], \Lambda \triangleq\left[\begin{array}{c}A \\ A^{2} \\ \vdots \\ A^{N}\end{array}\right]$.

The matrix $\Psi \in \mathbb{R}^{N \times N}$ used in (5) and (22) is obtained from the factorization $\Psi^{T} \Psi=\Phi^{T} \bar{Q} \Phi+\lambda I$.

\section{B. Proof of Theorem 3}

We will use ideas contained in [28], see also [21]. Equation (7) gives:

$$
\begin{aligned}
S_{x}\left(e^{j \omega}\right) & =G_{1}\left(e^{j \omega}\right) S_{b x}\left(e^{j \omega}\right)+G_{2}\left(e^{j \omega}\right) S_{w x}\left(e^{j \omega}\right) \\
S_{x b}\left(e^{j \omega}\right) & =G_{1}\left(e^{j \omega}\right) S_{b}\left(e^{j \omega}\right)+G_{2}\left(e^{j \omega}\right) S_{w b}\left(e^{j \omega}\right) \\
S_{x w}\left(e^{j \omega}\right) & =G_{1}\left(e^{j \omega}\right) S_{b w}\left(e^{j \omega}\right)+G_{2}\left(e^{j \omega}\right) S_{w}\left(e^{j \omega}\right) \\
S_{x n}\left(e^{j \omega}\right) & =G_{1}\left(e^{j \omega}\right) S_{b n}\left(e^{j \omega}\right)
\end{aligned}
$$

where we have used the fact that $\left\{w_{k}\right\}$ and $\left\{n_{k}\right\}$ are uncorrelated.

On the other hand, by conditioning upon the dropout process, expression (8) provides that:

$$
\begin{aligned}
R_{b x}(\ell) & =\mathcal{E}\left\{b_{k} x_{k+\ell}^{T}\right\}=p \mathcal{E}\left\{M b_{k-1} x_{k+\ell}^{T}\right\} \\
& +(1-p) \mathcal{E}\left\{\Psi^{-1}\left(\Gamma x_{k}+n_{k}\right) x_{k+\ell}^{T}\right\} \\
= & p M R_{b x}(\ell+1)+(1-p) \Psi^{-1}\left(\Gamma R_{x}(\ell)+R_{n x}(\ell)\right)
\end{aligned}
$$

By taking two-sided $z$-Transform, we obtain:

$$
S_{b x}\left(e^{j \omega}\right)=T\left(e^{j \omega}\right) \Psi^{-1}\left(\Gamma S_{x}\left(e^{j \omega}\right)+S_{n x}\left(e^{j \omega}\right)\right),
$$

where $T\left(e^{j \omega}\right)$ is as in (10).

Similarly, one can show that:

$$
\begin{aligned}
& S_{b n}\left(e^{j \omega}\right)=T\left(e^{j \omega}\right) \Psi^{-1}\left(\Gamma S_{x n}\left(e^{j \omega}\right)+S_{n}\left(e^{j \omega}\right)\right) \\
& S_{b w}\left(e^{j \omega}\right)=T\left(e^{j \omega}\right) \Psi^{-1} \Gamma S_{x w}\left(e^{j \omega}\right) .
\end{aligned}
$$

Substitution of (29) into (25) and of (26) into (28) gives:

$$
\begin{aligned}
S_{x w}\left(e^{j \omega}\right) & =H_{x}\left(e^{j \omega}\right) G_{2}\left(e^{j \omega}\right) S_{w}\left(e^{j \omega}\right) \\
S_{x n}\left(e^{j \omega}\right) & =H_{x}\left(e^{j \omega}\right) G_{1}\left(e^{j \omega}\right) T\left(e^{j \omega}\right) \Psi^{-1} S_{n}\left(e^{j \omega}\right) .
\end{aligned}
$$

If we now substitute (27) into (23), and after some algebraic manipulations, we obtain:

$$
\begin{array}{r}
S_{x}\left(e^{j \omega}\right)=H_{x}\left(e^{j \omega}\right) G_{1}\left(e^{j \omega}\right) T\left(e^{j \omega}\right) \Psi^{-1} S_{n x}\left(e^{j \omega}\right) \\
+H_{x}\left(e^{j \omega}\right) G_{2}\left(e^{j \omega}\right) S_{w x}\left(e^{j \omega}\right) .
\end{array}
$$

Finally, use of (30) and (31) in (32), gives (11).

To characterize $S_{b}\left(e^{j \omega}\right)$, we use (8) and write:

$$
\begin{aligned}
S_{b}\left(e^{j \omega}\right)=T\left(e^{j \omega}\right) & \Psi^{-1}\left(\Gamma S_{x b}\left(e^{j \omega}\right)+S_{n b}\left(e^{j \omega}\right)\right) \\
=T\left(e^{j \omega}\right) & \Psi^{-1}\left(\Gamma H_{x}\left(e^{j \omega}\right) G_{2}\left(e^{j \omega}\right) S_{b w}^{H}\left(e^{j \omega}\right)\right. \\
& \left.+\left(I+\Gamma H_{x}\left(e^{j \omega}\right) T\left(e^{j \omega}\right)\right) \Psi^{-1} S_{b n}^{H}\left(e^{j \omega}\right)\right),
\end{aligned}
$$

where we have utilized (24). Expressions (28)-(31), use of the Matrix Inversion Lemma, and some algebraic manipulations then provide the result (12).

Finally, (13) follows directly from (2).

\section{Proof of Theorem 4}

It follows from Theorem 1 , that $\vec{u}_{k}$ is obtained by nearest neighbour quantization of $\xi_{k}$ followed by an invertible transformation. Thus, from the data-processing inequality [29], the coding rate of $\vec{u}_{k}$ equals that of $\xi_{k}$.

The average code length of the quantized variables is given by the conditional entropy $H\left(q\left(\xi_{k}\right)\right) \triangleq H\left(q\left(\xi_{k}\right) \mid \eta_{k}\right)$ of the quantizer $q(\cdot)$, where the conditioning is with respect to the dither vector $\eta_{k}$. It follows from [25] that the coding rate (per $N$-block) of the quantizer is given by

$$
H\left(q\left(\xi_{k}\right)\right)=I\left(\xi_{k} ; \xi_{k}+n_{k}\right),
$$

where $I(\cdot, \cdot)$ denotes the mutual information.

Since we are using a memoryless quantizer (and, thus, a memoryless entropy coder), it follows from Fig. 2 and Eq. (33) that the coding rate in the NCS of Fig. 1 can be upper-bounded by the per-block mutual information over the equivalent noise channel from $\xi_{k}$ to $\xi_{k}+n_{k}$ as follows:

$$
\begin{aligned}
H\left(q\left(\xi_{k}\right)\right) & =I\left(\xi_{k} ; \xi_{k}+n_{k}\right) \\
& \stackrel{(a)}{\leq} I\left(\tilde{\xi}_{k} ; \tilde{\xi}_{k}+\tilde{n}_{k}\right)+\mathcal{D}\left(n_{k} \| \tilde{n}_{k}\right) \\
& \stackrel{(b)}{=} h\left(\tilde{\xi}_{k}+\tilde{n}_{k}\right)-h\left(\tilde{n}_{k}\right)+\mathcal{D}\left(n_{k} \| \tilde{n}_{k}\right) \\
& \stackrel{(c)}{=} \frac{1}{2} \log _{2}\left(\frac{\operatorname{det}\left(R_{\xi}(0)+R_{n}(0)\right)}{\operatorname{det} R_{n}(0)}\right)+\mathcal{D}\left(n_{k} \| \tilde{n}_{k}\right) \\
& \stackrel{(d)}{=} \frac{1}{2} \log _{2}\left(\operatorname{det}\left(I+\left(N / \sigma_{n}^{2}\right) R_{\xi}(0)\right)\right) \\
& \quad+\frac{N}{2} \log _{2}\left(2 \pi e \mathcal{G}\left(\Lambda_{N}\right)\right) .
\end{aligned}
$$


In the above, $h(\cdot)$ denotes differential entropy, $\mathcal{D}(\cdot \| \cdot)$ denotes Divergence (per dimension). The processes $\tilde{n}_{k}, \tilde{w}_{k}$ and $\tilde{\xi}_{k}$ are given by: ${ }^{7}$

$$
\begin{gathered}
\tilde{n}_{k} \sim \mathcal{N}\left(0, R_{n}(0)\right), \quad \tilde{w}_{k} \sim \mathcal{N}\left(0, R_{w}(0)\right), \\
\tilde{\xi}_{k}=\Gamma F_{w}(z) \tilde{w}_{k}+\Gamma F_{n}(z)\left(\tilde{\xi}_{k}+\tilde{n}_{k}\right) .
\end{gathered}
$$

The bound (a) follows from Lemma 2 in [30], (b) follows by writing the mutual information in terms of differential entropies and noticing that $\tilde{n}_{k}$ is independent of $\tilde{\xi}_{k}$, and (c) follows from the definition of the differential entropy of a Gaussian variable [29]. Finally, $(d)$ follows from the properties of an ECDQ [26].

To obtain the bound (20), we recall that, due to possible inefficiency of an entropy coder, the actual coding rate satisfies [29]:

$$
H\left(q\left(\xi_{k}\right)\right) \leq R \leq H\left(q\left(\xi_{k}\right)\right)+1 .
$$

The theorem follows from noting that $\mathcal{G}\left(\Lambda_{N}\right) \leq 1 / 12$ for any lattice [27].

\section{Proof of Lemma 2}

We first note that, due to feedback and $d, n$ and $w$ being i.i.d., the signals $x, \vec{u}, u$ and $b$ are stationary if and only if $\xi$ is stationary. By assumption $\xi$ is stationary. Thus, (4), (21) and (19) provide:

$$
R_{\xi}(0)=\Gamma R_{x}(0) \Gamma^{T}=\frac{\operatorname{det} R_{\xi}(0)}{\gamma N} \Gamma M_{n} \Gamma^{T}+\Gamma M_{w} \Gamma^{T} .
$$

Since the term $\Gamma M_{w} \Gamma^{T}$ in the above expression is positive semi-definite, we require:

$$
R_{\xi}(0)-\frac{\operatorname{det} R_{\xi}(0)}{\gamma N} \Gamma M_{n} \Gamma^{T} \succeq 0 .
$$

Solving for $\gamma$ gives the condition:

$$
\gamma I-\frac{\operatorname{det} R_{\xi}(0)}{N} \Gamma M_{n} \Gamma^{T} R_{\xi}^{-1}(0) \succeq 0 .
$$

By definition, a matrix is positive semidefinite if and only if all its eigenvalues are non-negative. The result follows from noting that the eigenvalues of

$$
\gamma I-\frac{\operatorname{det} R_{\xi}(0)}{N} \Gamma M_{n} \Gamma^{T} R_{\xi}^{-1}(0)
$$

are $\left\{\gamma-\nu_{i}\right\}$.

\section{REFERENCES}

[1] J. P. Hespanha, P. Naghshtabrizi, and Y. Xu, "A survey of recent results in networked control systems," Proc. IEEE, vol. 1, pp. 138-162, Jan. 2007.

[2] G. N. Nair, F. Fagnani, S. Zampieri, and R. J. Evans, "Feedback control under data rate constraints: An overview," Proc. IEEE, vol. 95, pp. 108-137, Jan. 2007.

[3] L. Schenato, B. Sinopoli, M. Franceschetti, K. Poolla, and S. S. Sastry, "Foundations of control and estimation over lossy networks," Proc. IEEE, vol. 95, pp. 163-187, Jan. 2007.

[4] J. R. Moyne and D. M. Tilbury, "The emergence of industrial control networks for manufacturing control, diagnostics, and safety data," Proc. IEEE, vol. 95, pp. 29-47, Jan. 2007.

\footnotetext{
${ }^{7}$ Note that $R_{\tilde{\xi}}(0)=R_{\xi}(0)$.
}

[5] D. Hristu-Varsakelis and W. S. Levine, eds., Handbook of Networked and Embedded Systems. Boston, MA: Birkhäuser, 2005.

[6] A. Bemporad, "Predictive control of teleoperated constrained systems with unbounded communication delays," in Proc. IEEE Conf. Decis. Contr., (Tampa, Florida), pp. 2133-2138, 1998.

[7] A. Casavola, E. Mosca, and M. Papini, "Predictive teleoperation of constrained dynamic systems via internet-like channels," IEEE Trans. Contr. Syst. Technol., vol. 14, pp. 681-694, July 2006.

[8] P. L. Tang and C. W. de Silva, "Stability validation of a constrained model predictive networked control system with future input buffering," Int. J. Contr., vol. 80, pp. 1954-1970, Dec. 2007.

[9] G. P. Liu, J. X. Mu, D. Rees, and S. C. Chai, "Design and stability analysis of networked control systems with random communication time delay using the modified MPC," Int. J. Contr., vol. 79, pp. 288297, Apr. 2006.

[10] Y.-B. Zhao, G. P. Liu, and D. Rees, "Improved predictive control approach to networked control systems," IET Control Theory Appl., vol. 2, no. 8, pp. 675-681, 2008.

[11] G. Pin and T. Parisini, "Stabilization of networked control systems by nonlinear model predictive control: A set invariance approach," in Int. Workshop on Assessment and Future Directions of NMPC, 2008.

[12] D. E. Quevedo, E. I. Silva, and G. C. Goodwin, "Packetized predictive control over erasure channels," in Proc. Amer. Contr. Conf., (New York, N.Y.), 2007.

[13] R. Findesisen and P. Varutti, "Stabilizing nonlinear predictive control over nondeterministic networks," in Int. Workshop on Assessment and Future Directions of NMPC, 2008.

[14] D. E. Quevedo, E. I. Silva, and G. C. Goodwin, "Control over unreliable networks affected by packet erasures and variable transmission delays," IEEE J. Select. Areas Commun., vol. 26, pp. 672-685, May 2008.

[15] O. C. Imer, S. Yüksel, and T. Bassar, "Optimal control of LTI systems over unreliable communication links," Automatica, vol. 42, pp. 14291439, Sept. 2006.

[16] J. Wu and T. Chen, "Design of networked control systems with packet dropouts," IEEE Trans. Automat. Contr., vol. 52, pp. 1314-1319, July 2007.

[17] D. E. Quevedo, G. C. Goodwin, and J. A. De Doná, "Finite constraint set receding horizon quadratic control," Int. J. Robust Nonlin. Contr., vol. 14, pp. 355-377, Mar. 2004.

[18] A. Gersho and R. M. Gray, Vector Quantization and Signal Compression. Boston, MA: Kluwer Academic, 1992.

[19] R. Zamir, Y. Kochman, and U. Erez, "Achieving the Gaussian ratedistortion function by prediction," IEEE Trans. Inform. Theory, vol. 54, pp. 3354-3364, July 2008.

[20] G. C. Goodwin, D. E. Quevedo, and E. I. Silva, "Architectures and coder design for networked control systems," Automatica, vol. 44, pp. 248-257, Jan. 2008.

[21] E. I. Silva, D. E. Quevedo, and G. C. Goodwin, "Optimal coding for bit-rate limited networked control systems in the presence of data loss," in Proc. IEEE Conf. Decis. Contr., (New Orleans, LA), pp. 665-670, 2007.

[22] M. S. Derpich, E. I. Silva, D. E. Quevedo, and G. C. Goodwin, "On optimal perfect reconstruction feedback quantizers," IEEE Trans. Signal Processing, vol. 56, pp. 3871-3890, Aug. 2008.

[23] O. L. V. Costa, M. D. Fragoso, and R. P. Marques, Discrete-Time Markov Jump Linear Systems. London, U.K.: Springer-Verlag, 2005.

[24] E. Agrell, T. Eriksson, A. Vardy, and K. Zeger, "Closest point search in lattices," IEEE Trans. Inform. Theory, vol. 48, pp. 2201-2214, Aug. 2002.

[25] R. Zamir and M. Feder, "On universal quantization by randomized uniform/lattice quantizers," IEEE Trans. Inform. Theory, vol. 38, pp. 428-436, Mar. 1992.

[26] R. Zamir and M. Feder, "On lattice quantization noise," IEEE Trans. Inform. Theory, vol. 42, pp. 1152-1159, July 1996.

[27] J. H. Conway and N. J. A. Sloane, Sphere packings, lattices and groups. London, U.K.: Springer-Verlag, 1988.

[28] Q. Ling and M. D. Lemmon, "Power spectral analysis of networked control systems with data dropouts," IEEE Trans. Automat. Contr., vol. 49, pp. 955-960, June 2004.

[29] T. M. Cover and J. A. Thomas, Elements of Information Theory. Hoboken, New Jersey: Wiley-Interscience, second ed., 2006.

[30] M. S. Derpich, J. Østergaard, and D. E. Quevedo, "Achieving the quadratic Gaussian rate-distortion function for source uncorrelated distortions." http://arxiv.org/pdf/0801.1718, July 2008. 